\title{
Stress, Burnout, Social Support and General Health of Nurses in Intensive Care Unit
}

\author{
Pawan Rathi ${ }^{1}$, Nishant Ohri ${ }^{2}$, Srikanth Reddy ${ }^{3}$ Aditya Dubey ${ }^{4}$, Amandeep Gill ${ }^{5}$, Aditi Patel ${ }^{6}$ \\ ${ }^{1}$ Associate Professor \& Head of Department \\ ${ }^{2,5}$ Senior Resident \\ 3,6 Junior Resident \\ ${ }^{4}$ Associate Professor \\ Department of Psychiatry, Sri Aurobindo Medical College \& P.G. Institute, Indore \\ Corresponding author: Nishant Ohri \\ Email-nishantohri492@gmail.com
}

\begin{abstract}
Background and Objectives: Nurses working in the intensive care unit (ICU) may be exposed to considerable stress. Gaps in research focusing on stress, burnout, social support and general health of nurses is evident within developing contexts like India. The study aim was to assess the level of and the relationship between (1) stress, (2) burnout, (3) social support, \& (4) general health of nurses working in intensive care units.

Methods: A cross-sectional study was performed at ICUs at tertiary care hospitals, Indore. The self report questionnaire consisted of a demographic questionnaire, The Multidimensional scale of Perceived Social Support, The Abbreviated Maslach Burnout Inventory, the Perceived Stress Scale, and lastly the General Health Questionnaire. 115 of $186(62 \%)$ answered the questionnaire.

Results: The mean age of study subjects was 27 years $(\mathrm{SD}=4.85 ; \mathrm{n}=111)$. Out of the total 71 were males, with mean age of 27.7 years, and 40 females with the mean age of 26.25 years. Mean Burnout score of total study population was $30.27(\mathrm{SD}=11.09 ; \mathrm{N}=111)$. No significant difference in mean burnout scores of males and females was found $(\mathrm{p}=0.960 ; \mathrm{t}=0.0502 ; \mathrm{df}=110)$. One-way ANOVA was done taking Burnout as a factor with 3 levels: Higher mean GHQ-12 scores were seen in groups with higher burnout severity $[\mathrm{F}(2,108)$ $=13.470 ; \mathrm{p}<0.05]$. GHQ-12 scores better predicted burn out than perceived stress and social support. But, people with high perceived stress had significantly lower social support mean scores $[\mathrm{F}(2,108)=$ $6.17 ; p=0.0017]$. Correlation between stress and burnout was insignificant.

Conclusion: GHQ-12 scores better predicted burnout in ICU staff. Staff with higher stress levels had poorer social-support.
\end{abstract}

Keywords: Depression, anxiety, stress, medical interns.

(Paper received $-9^{\text {th }}$ November 2018 , Peer review completed $-10^{\text {th }}$ December 2018 )

(Accepted $-14^{\text {th }}$ December 2018)

\section{INTRODUCTION}

The critical care nurse has always been considered to be having one of the most taxing of job profiles. Patient management protocols are strict, time-bound and demand attention and efficiency. Working hours may be long, working shifts change often and patient monitoring requires constant vigilance. With stress, weather work related or personal, and poor coping skills burnout in nurses maybe anticipated to be high [1]. Studying burnout in such environments is relevant. Burnout can be seen as a psychological experience attitudes, motives and expectations and my result in feelings of overburden, feelings of lacking agency and lacking meaningful connections. Maslach considered emotional exhaustion, depersonalization and personal accomplishment to be the defining characteristics of burnout [2]. 
High levels of burnout are not only deleterious to the subject but also to the organization as a whole [3]. High frequency of absenteeism, decreased institutional turnover, poor patients' satisfaction and confrontational attitude towards patient caregivers have been shown to be related to burnout [4]. Poor patient outcome is another important concern related to burnout as it directly affects patient-care [5]. On the other hand, institutional limitations themselves maybe factors that lead to poor job satisfaction and eventually high burnout. The mismatch between job expectation and actual working environment frequently results in poor job satisfaction [6].

Personal stress and personality too play an important role in predicting burnout. High neurotic traits, as anticipated, have been shown to contribute to stress and burnout after controlling for factors like age, working hours and total duration of work. High conscientiousness has also been shown to have a stress aggravating effect [7].

In the present study, we have considered the role of personal stress, social support, and general health in predicting burnout in the intensive care unit staff. As per the evidence from previous studies, we hypothesize that high stress and poor general health may be associated with high burnout while good social support may have a protective effect. The study aimed to assess scores of personal stress, perceived social support, general health and burnout using appropriate questionnaires and to analyse the extent to which the variance in burnout scores is explained by age, stress, social support and general health.

\section{METHODOLOGY}

A total of 136 ICU nurses in different hospitals in Indore city were approached for a cross sectional survey of burnout, perceived stress, perceived social support and general health. Out of these, 111 subjects responded to the questionnaires. Maslach Burnout Inventory - Human Services Survey (MBI-HSS) [8] was used to grade the severity of burnout syndrome. The Perceived Stress Scale [9] was used to assess personal stress. For quantifying social support, the Multidimensional scale of Perceived Social Support [10] was used and the General Heath Questionnaire-12 (GHQ-12) [11] was used to assess physical and mental wellbeing. The subjects were matched for their demographic variables. The variables that showed significant difference between burnouts and non-burnouts were considered for one-way ANNOVA to analyse the variance contributed by them. One-way ANNOVA was also applied to stress, social support and GHQ12 scores keeping burnout scores as a factor at three levels.

\section{RESULTS}

In all we recruited 111 subjects out of which 71 were male and 40 , female. The mean age of subjects in the study was 27 years $(\mathrm{SD}=4.85)$ and the mean burnout score was $50.69(\mathrm{SD}=20.35)$, with no significant difference in the burnout scores between male and female subjects $(\mathrm{p}=0.960 ; \mathrm{t}=0.0502 ; \mathrm{df}=110)$. The mean scores of other measures are mentioned in table 1. Depending on approximately one standard deviation subjects were categorized into having no/low burnouts (scores 0 to $40, n=49$ ), medium ( 41 to $60 ; n=34$ ) and high burnouts ( 61 or more; $n=28$ ).

Table 1 - Scores on various scales used in the study

\begin{tabular}{|llll|} 
Scale & Mean & SD & Range \\
\hline Maslach Burnout Inventory & 50.69 & 20.35 & $2-84$ \\
GHQ-12 & 4.98 & 2.40 & $0-11$ \\
Perceived Social Support & 5.08 & 1.37 & $1-6.92$ \\
Perceived Stress & 19.65 & 5.31 & $1-30$ \\
\hline
\end{tabular}

One way ANOVA was done taking burnout as the factor (with 3 levels of severity) against GHQ-12, Perceived Stress and Perceived Social Support scores. In the case of GHQ-12, low burnouts had the mean score of 4, medium burnouts scored a mean of 4.88 and high burnouts scored 6.67 (Table 2). The differences in the GHQ-12 scores between the categories were highly significant $[F(2,108)=13.470 ; p<0.00001]$. The 
differences mean GHQ-12 scores at three levels of perceived stress were insignificant $[F(2,108)=1.084$; $\mathrm{p}=0.341]$.

Table 2 - Burnout and GHQ scores

\begin{tabular}{|ccccc|}
\hline GHQ scores of & $\begin{array}{c}\text { GHQ scores of } \\
\text { low burnouts }\end{array}$ & $\begin{array}{c}\text { GHQ scores of } \\
\text { medium burnouts }\end{array}$ & Total \\
High burnouts & \\
\hline Mean & 49 & 34 & 28 & 111 \\
\hline Std.Dev. & 4 & 4.8824 & 6.6786 & 4.9375 \\
\hline & 1.9378 & 2.4091 & 2.3262 & 2.4209 \\
\hline
\end{tabular}

Mean perceived stress scores at the three burnout levels were not significantly different (table 3 ) from each other $[\mathrm{F}(2,108)=1.19595 ; \mathrm{p}=0.306]$; also the correlation between them was weak and insignificant $[\mathrm{R}=$ 0.054; $\mathrm{p}=0,574]$. Among the different domains of Burnout, high personal accomplishment correlated with low perceived stress $(\mathrm{R}=-0.217 ; \mathrm{p}=0.02)$. Other domains showed no correlation.

Table 3 - Burnout levels and difference in mean Perceived Stress Scores

\begin{tabular}{|ccccc|}
\hline Stress scores of & $\begin{array}{c}\text { Stress scores of } \\
\text { low burnouts }\end{array}$ & $\begin{array}{c}\text { Stress scores of } \\
\text { medium burnouts }\end{array}$ & Total \\
\hline $\mathbf{N}$ & 49 & 34 & 28 & 111 \\
\hline Mean & 20.0408 & 20.2059 & 18.3214 & 19.6577 \\
\hline Std.Dev. & 4.8689 & 5.2845 & 6.0373 & 5.3181 \\
\hline
\end{tabular}

The mean Perceived social support score was 5.08 ( $\mathrm{SD}=1.37$ ), which falls in 'high' social support category. Only 12 out of 111 subjects fell in the low social support category range between 1 and 2.9. Social support scores were also tested against perceived stress (Table 4) at three levels of stress (low, moderate and high). Mean social support score of subjects who perceived high levels of stress was significantly low (3.64) compared to those who perceived low or moderate stress $(5.21$ and $5.22 ; \mathrm{F}(2,108)=6.706 ; \mathrm{p}=0.0017)$. Social Support scores and GHQ-12 scores also showed a significant positive correlation $(\mathrm{R}=+0.337 ; \mathrm{p}<000.1)$ (Table 6).

Table 4 - Relation between perceived stress and perceived social support

\begin{tabular}{|ccccc|}
\hline & $\begin{array}{c}\text { Social support scores of } \\
\text { low stress levels }\end{array}$ & $\begin{array}{c}\text { Social support scores of } \\
\text { medium stress levels }\end{array}$ & $\begin{array}{c}\text { Social support scores of } \\
\text { High stress levels }\end{array}$ & Total \\
\hline $\mathbf{N}$ & 12 & 89 & 10 & 111 \\
\hline Mean & 5.21 & 5.22 & 3.64 & 5.0827 \\
\hline Std.Dev. & 1.68 & 1.26 & 1.15 & 1.3704 \\
\hline
\end{tabular}

Social support scores of high burnouts were higher compared with scores of low and medium burnouts $[\mathrm{F}(2,108)=10.07 ; \mathrm{p}=0.00009]$. Burnout domains of depersonalization and emotional exhaustion, both had significant positive correlation with perceived social support $(+0.347$ and +0.440 , respectively; $p<0.0001$ ) (Table 7). Domains of satisfaction with work and personal accomplishment did not show any correlation with perceived social support.

\section{DISCUSSION}

The aim of our study was to examine the effect of demographic variables, perceived Stress, general health and social support on burnout. We had hypothesized that older age, high perceived stress, low social support and poor general heath should predict high burnout. 
The age of our subjects explained the variations in burnout and perceived stress scores significantly better than variations in GHQ12 and social support scores. The more the age the chances of burnout were higher, but perceived stress was the highest in mid-range of age ( 25 to 29 years). At the same time, the variations at different levels of age were more discreet in regard to burnout $(F$-ratio $=175.56)$ than perceived stress $(\mathrm{F}=4.52)$ (Table 5). Explained by more stressful life events in this age group? Correlation of increasing age with burnout has been reproduced in numerous studies. A study from Pune that recruited 298 nurses also found a negative correlation between age and burnout scores. The study did not analyze the correlation between stress and burnout, but like our study, found nurses of the mid age-range to be having the highest stress [12].

Table 5 - Stress scores at different age levels

\begin{tabular}{|ccccc|}
\hline & $\mathbf{2 1}-\mathbf{2 4}$ Years & $\mathbf{2 5}-\mathbf{2 9}$ Years & $\mathbf{3 0}$ and above & Total \\
\hline $\mathbf{N}$ & 38 & 51 & 22 & 111 \\
\hline Mean & 18.9737 & 21.1373 & 17.4091 & 19.6577 \\
\hline Std.Dev. & 4.9728 & 4.7708 & 6.2386 & 5.3181 \\
\hline
\end{tabular}

When burnout was taken as factor at three levels of severity against perceived stress, the differences in mean stress scores were not significant, but numerically, 'high burnouts' had lowest stress scores. In a study from Johannesburg that recruited 895 nurses, personal stress accounted for $27 \%$ variance in burnout scores [13]. Although we found no correlation between stress and burnout, but the personal accomplishment domain had a significantly negative correlation with stress, suggesting that with more personal accomplishment stress was low. Noha Elshaer and colleagues, analyzed job related stress against burnout domains and found significantly higher scores of emotional exhaustion in subjects who suffered from intragroup conflicts, and low emotional exhaustion in subjects who assumed responsibility for people's lives, who had job satisfaction and perceived control [14].

We had initially hypothesized a relationship between burnout and perceived stress, but found none. One possible reason could be that our high burnouts fell in the upper age-range, while the same age range had the least perceived stress, so it is not surprising that burnout and perceived stress had weak and poor correlation. Subjects in the mid age range of 25 to 29 years had the highest perceived stress. High Burnouts had higher GHQ12 scores, but at that same time, surprisingly they had higher perceived social support too. Perceived social support, curiously, also had a strong positive and significant correlation with GHQ12 scores. In short, social support was significantly higher in High-burnouts and in subjects with higher GHQ12 scores (poor general health). High perceived stress on the other hand, predicted low social support.

Table 6 - Correlation between the scales

\begin{tabular}{|cccc|}
\hline & $\begin{array}{c}\text { Correl. } \\
\text { Coeff. }\end{array}$ & P value & N \\
\hline Burnout Vs. GHQ12 & 0.478 & $<0.0001$ & 111 \\
\hline Social Sup. Vs. GHQ12 & 0.337 & $<0.0001$ & 111 \\
\hline Burnout Vs. Social Supp. & 0.434 & $<0.0001$ & 111 \\
\hline
\end{tabular}

Numerous studies have confirmed association between burnout and poor physical/mental health [15-17]. In another study on 1200 nurses in Johannesburg, Natasha Khamisa and her team found that emotional exhaustion and depersonalization domains of burnout $31 \%$ variance in anxiety/insomnia and $21 \%$ variance in somatic symptoms. The study also observed that work stress (especially related to safety of nurses) explained $11 \%$ of variance in social dysfunction [18].

In our study social support was uniform and high across the three levels of age, with a high overall average of 5.08, suggesting a negative skew (mode $=6.50$; Skew $=-0.86$ ). This should explain why social support is showing disproportionately strong positive correlations. Also, since we did not screen for specific disorders it will be wrong to assume that all those who scored high on GHQ-12 fulfilled the full criteria for specific 
disorders (like Major depression, anxiety etc.). Two domains of Burnout: Depersonalization and Emotional Exhaustion, contributed most towards significant positive correlation with social support. The same domains also contributed the most towards a positive correlation with psychological morbidity. So, it is plausible that subjects with higher emotional exhaustion and depersonalization pursued social support more, and got it.

Table 7 - Correlation of Emotional Exhaustion and Depersonalization

\begin{tabular}{|ccc|}
\hline & Vs. GHQ-12 & Vs. Per. Social Supp. \\
\hline Emotional Exhaustion & $\mathrm{R}=0.495 ; \mathrm{p}<0.0001$ & $\mathrm{R}=0.440 ; \mathrm{p}<0.0001$ \\
\hline Depersonalization & $\mathrm{R}=0.497 ; \mathrm{p}<0.0001$ & $\mathrm{R}=0.347 ; \mathrm{p}<0.0001$ \\
\hline
\end{tabular}

\section{Limitations}

Duration of service is an important factor that we did not look deeper into in our study. Various studies have suggested that duration of service and working hours may be important factors in predicting burnout; but many studies have found no correlation as well. Skewed perceived social support scores may have been responsible for significant positive correlation with burnout. Applying nonparametric tests may have led to more robust result in this context.

\section{CONCLUSION}

Perceived stress does not predict burnout, except personal accomplishment, which is negatively correlated with stress. Highly stressed subjects had significantly poor social support. But perceived stress and GHQ12 scores were not related. High GHQ12 scores predict burnout, especially in emotional exhaustion and depersonalization domains. On the other hand, high burnouts and subjects with high GHQ12 scores perceived good social support. Only $9 \%$ of subjects reported low social support.

\section{REFERENCES}

1. Kalliath T, Morris R. Job satisfaction among nurses: A predictor of burnout levels. J Nurs Adm 2002;32:64851.

2. Leiter MP, Robichaud L. Relationships of occupational hazards with burnout: an assessment of measures and models. J Occup Health Psychol 1997;2(1):35-44.

3. King N. Recognition of work-related diseases: an original collaborative project in Québec. New Solut 1999;9(4):427-37.

4. Duquette A, Kérouac S, Sandhu BK, Beaudet L. Factors related to nursing burnout: a review of empirical knowledge. Issues Ment Health Nurs 1994;15(4):337-58.

5. Gibson D. The gaps in the gaze in South African hospitals. Soc Sci Med. 2004;59:2013-24.

6. Spector PE. Job Satisfaction: Application, Assessment, Causes and Consequences. Sage Publications. 1997.

7. Piedmont RL. A longitudinal analysis of burnout in the health care setting: the role of personal dispositions. J Pers Assess 1993;61(3):457-73.

8. Maslach C, Jackson S. Maslach Burnout Inventory Manual. 2nd edition. Palo Alto, CA: Consulting Psychologists Press, 1986.

9. Cohen S, Kamarck T, Mermelstein R. A global measure of perceived stress. J Health Soc Behav 1983;1:38596.

10. Zimet GD, Dahlem NW, Zimet SG, Farley GK. The multidimensional scale of perceived social support. J Personal Assess 1988;52(1):30-41.

11. Pevalin DJ. Multiple applications of the GHQ-12 in a general population sample: an investigation of long-term retest effects. Soc Psych Psychiatr Epidemiol 2000;35(11):508-12.

12. Divinakumar KJ, Pookala SB, Das RC. Perceived Stress, psychological wellbeing and burnout among female nurses working in government hospitals. Int J Res Med Sci 2014;2(4):1511-5.

13. Khamisa N, Peltzer K, Ilic D, Oldenburg B. Effect of personal and work stress on burnout, job satisfaction and general health of hospital nurses in South Africa. Health SA Gesondheid 2017. 22(12):252-8.

14. Elshaer NS, Moustafa MS, Aiad MW, Ramadan MI. Job stress and burnout syndrome among critical care healthcare workers. Alexandria J Med 2018;54(3):273-7. 
15. McKee, M.G.; Ashton, K. Stresses of daily life. In Clinical Preventive Medicine, 3rd ed.; Lang, R., Hensrud, D., Eds.; AMA Press: Chicago, IL, USA, 2004.

16. Penson RT, Dignan FL, Canellos GP, Picard CL, Lynch TJ. Burnout: caring for the caregivers. The Oncol 2000;5(5):425-34.

17. Weber A, Jaekel-Reinhard A. Burnout syndrome: a disease of modern societies?. Occup Med 2000;50(7):5127.

18. Khamisa N, Oldenburg B, Peltzer K, Ilic D. Work related stress, burnout, job satisfaction and general health of nurses. Int J Environ Res Pub Health 2015;12(1):652-66.

\section{Acknowledgements - Nil \\ Conflict of Interest - Nil \\ Funding - Nil}

